\title{
A NECESSARY AND SUFFICIENT CONDITION FOR MEMBERSHIP IN [uv]
}

\author{
D. G. MEAD
}

Levi has obtained [1] for $\left[y^{p}\right]$ and $[u v]$ sufficiency conditions for membership of a power product in the ideal, which tests membership, in certain cases, by a calculation using only the weight and degree of the pp. In this paper we show Levi's conditions for [uv] are necessary as well as sufficient, in contradistinction to $\left[y^{p}\right]$ (see [2]). (This, of course, will show that the answer to Ritt's question [5, p. 177] "What is the least power of $u_{i} v_{j}$ which is in [uv]?" is $i+j+1$.)

Levi's sufficiency condition can be stated in the following manner: If the pp. $P$ has a negative number in its weight sequence, $P \in[u v]$. Since it is known [2, Theorem III, p. 426] that one need only consider pp. with zero excess weight, for the necessity it will suffice to prove the

THEOREM. If the pp. $P$ has zero excess weight and a non-negative weight sequence, $P \notin[u v]$.

We recall some of the definitions of [1] and [2] as well as introduce some notation for this paper.

Let $U(i, r, k)$ represent the product $u_{i_{1}+r} u_{i_{2}+r} \cdots u_{i_{k}+r}$. The signature of $P=U(i, 0, m) V(j, 0, n)$ is $(m, n)$ and the weight of $P$ is $\sum i_{\alpha}$ $+\sum j_{\beta}$. For all possible pairs $\left(m^{\prime}, n^{\prime}\right)$ where $1 \leqq m^{\prime} \leqq m, 1 \leqq n^{\prime} \leqq n$, we consider the weight of a factor of $P$ of least weight and signature $\left(m^{\prime}, n^{\prime}\right)$, minus $m^{\prime} n^{\prime}$. This set of numbers we call the weight sequence of $P$. If all the numbers of the weight sequence are non-negative, we say that $P$ has a non-negative weight sequence. The weight of $P$ minus $m n$ is called the excess weight of $P$.

We facilitate our work by introducing the new variables $\bar{u}_{i}=u_{i} / i$ ! and $\bar{v}_{j}=v_{j} / j$ !. For these variables, we have $\left(\bar{u}_{i}\right)^{\prime}=(i+1) \bar{u}_{i+1}$ and $\left(\bar{v}_{j}\right)^{\prime}=(j+1) \bar{v}_{j+1}$. To simplify the notation, we write $u_{i}, v_{j}$ for $\bar{u}_{i}, \bar{v}_{j}$ respectively.

If $P$ is a pp. of signature $(m, n)$ and of zero excess weight, then ${ }^{1}$ $P \equiv c u^{m} v_{m}^{n}$, and, calling $c$ the multiplier of $P$, we write $m(P)=c$. Finally, let $D^{k}=\partial^{k} /\left(\partial v_{i_{1}} \partial v_{i_{2}} \cdots \partial v_{i_{k}}\right)$.

Lemma I. Assume the pp. $A$ is of zero excess weight and is free of $v_{0}$. If $A=u_{k} P\left(u, v_{1}\right)$, where $P(u, v)$ is a pp. in $u$ and $v$, then

Received by the editors October 31, 1964.

1 Use [1, Theorem 1.1, p. 543] with the fact that there is a unique $\alpha$ term of the same signature and weight as $P$. 


$$
k ! m\left(u_{k} P\left(u, v_{1}\right)\right)=(-1)^{k} \sum m\left(V(i, 1, k) D^{k} P(u, v)\right)
$$

where the summation is over all ordered sets, $\left(i_{1}, \cdots, i_{k}\right)$, of non-negative integers.

Proof. First we note the equation is meaningful since every term does have zero excess weight. It has been pointed out, [4], that the proof given in [2, Lemma I, p. 429] can be used to show that if $U(i, 0, m) V(j, 0, n)$ has zero excess weight, $m(U(i, 0, m) V(j, 0, n))$ $=m\left(u_{0} U(i, 0, m) V(j, 1, n)\right) .{ }^{2}$ This constitutes the statement of our lemma for $k=0$. We proceed with the proof using induction on $k$. Let $A=u_{k+1} P\left(u, v_{1}\right)$ have zero excess weight. Both $u_{k} P\left(u, v_{1}\right)$ and $V(i, 1, k) D^{k} P(u, v)$ are in $[u v]$. Thus ${ }^{3}$

$$
k ! u_{k} P\left(u, v_{1}\right) \equiv(-1)^{k} \sum V(i, 1, k) D^{k} P(u, v)
$$

and, taking derivatives of both sides

$$
\begin{aligned}
&(k+1) ! u_{k+1} P\left(u, v_{1}\right)+k ! u_{k} \sum u_{j}^{\prime} \partial P\left(u, v_{1}\right) / \partial u_{j}+k ! u_{k} \sum v_{j}^{\prime} \partial P\left(u, v_{1}\right) / \partial v_{j} \\
& \equiv(-1)^{k} \sum V(i, 1, k) u_{j}^{\prime} \partial D^{k} P(u, v) / \partial u_{j} \\
&+(-1)^{k} \sum V(i, 1, k) v_{j}^{\prime} \partial D^{k} P(u, v) / \partial v_{j} \\
&+(-1)^{k} \sum(V(i, 1, k))^{\prime} D^{k} P(u, v) .
\end{aligned}
$$

By the induction hypothesis, the multipliers ${ }^{4}$ of the first sums on the two sides of the congruence are equal and the multiplier of the second sum of the left equals

$$
\begin{aligned}
(-1)^{k} m & \sum k V(i, 1, k-1) v_{i_{k}+1}\left(i_{k}+1\right) D^{k-1} \partial P(u, v) / \partial v_{i_{k}-1} \\
& +(-1)^{k} m \sum V(i, 1, k)(j+1) v_{j} D^{k} \partial P(u, v) / \partial v_{j-1} \\
& =(-1)^{k} m \sum k V(i, 1, k-1) v_{i_{k}+2}\left(i_{k}+2\right) D^{k} P(u, v) \\
& +(-1)^{k} m \sum V(i, 1, k)\left(i_{k+1}+2\right) v_{i_{k+1}+1} D^{k+1} P(u, v) .
\end{aligned}
$$

Finally, since the second sum on the right equals

$$
(-1)^{k} \sum\left(i_{k+1}+1\right) V(i, 1, k+1) D^{k+1} P(u, v)
$$

2 The referee supplied the following alternative proof. Let $h$ be the isomorphism of $F\left[u_{0}, u_{1}, \cdots ; v_{0}, v_{1}, \cdots\right]$ obtained by raising each subscript on a $v$ by 1 and let $h[u v]$ stand for the image of $[u v]=\left(u v, u_{1} v+u v_{1}, u_{2} v+u_{1} v_{1}+u v_{2}, \cdots\right)$ under this isomorphism. Then, obviously, $u h[u v] \subset[u v]$. Let $U V \equiv c u^{m} v_{m}^{n}[u v]$. Then $U h(V)$ $\equiv c u^{m} v_{m+1}^{n}(h[u v])$, whence $u U h(V) \equiv c u^{m+1} v_{m+1}^{n}[u v]$, Q.E.D.

${ }^{3}$ Throughout the proof of Lemma I, each summation is over all ordered sets of nonnegative integers of the symbols $i \hat{\alpha}$ and $j \hat{\beta}$ which appear in the terms being summed.

${ }^{4}$ The multiplier of a sum of terms is defined to be the sum of the multipliers of the individual terms. 
and the third sum on the right equals

$$
(-1)^{k} \sum k V(i, 1, k-1)\left(i_{k}+2\right) v_{i_{k}+2} D^{k}(P u, v) \text {, }
$$

we have

$$
\begin{gathered}
(k+1) ! m\left(u_{k+1} P\left(u, v_{1}\right)\right)+(-1)^{k} m \sum\left(i_{k+1}+2\right) V(i, 1, k+1) D^{k+1} P(w, v) \\
=(-1)^{k} m \sum\left(i_{k+1}+1\right) V(i, 1, k+1) D^{k+1} P(u, v)
\end{gathered}
$$

which completes the proof.

Lemma II. If the $p p$. $A$, of signature $\left(d_{1}, d_{2}\right)$, has a non-negative weight sequence and zero excess weight, $A \equiv(-1)^{t} c u^{d_{1}} v_{d_{1}}^{d_{2}}$, where $t$ is the $u$-weight of $A$ and $c>0$.

Proof. If $d_{1}=1, A$ is either $u v_{1}^{d_{2}}$ or $u_{1} v v_{1}^{d_{2}-1}$, and in each case the lemma is easily seen to be true. We complete the proof employing induction on $d_{1}$.

We assume for the moment that $A$ is free of $v_{0}$ and use Lemma I, noting that every term on the right side of the congruence has $u$ degree $d_{1}-1$ and $u$-weight $k$ less than the $u$-weight of $A$. Thus there is no cancellation among the numbers on the right, as either the induction hypothesis applies or the pp. has a negative term in its weight sequence and, being in $[u v]$, its multiplier is zero.

The proof of this case ( $A$ free of $v_{0}$ ) will be complete once we produce a pp. on the right side of the congruence with a non-negative weight sequence. If the $v$-factor of $A$ is $v_{a_{1}+1} v_{a_{2}+1} \cdots v_{a_{2}+1},\left(a_{1} \leqq a_{2}\right.$ $\left.\leqq \cdots \leqq a_{d_{2}}\right)$ such a term is

$$
Q=V(a, 1, k) \partial^{k} P(u, v) /\left(\partial v_{a_{1}} \partial v_{a_{2}} \cdots \partial v_{a_{k}}\right) .
$$

Assume this false and let $S$ be a factor of $Q$ with negative excess weight. If we can show that $S$ involves a $v_{j}$ with $j \geqq a_{k}+1$, we may assume, without loss of generality, that $S$ is a multiple of $V(a, 1, k)$. Since $V(a, 1, k)$ is a factor of $A$, we see that $S$ must involve some $v_{j}$ from the $k$ th partial derivative. Assume $S=U V$ has $u$-degree $=b$, $u$-weight $=w_{u}$ and involves only $v_{j}$ with $j \leqq a_{k}$. Then $a_{k+1}=a_{k}$ and we define $r, s$, and $e$ by $a_{r}<a_{r+1}=a_{k}=a_{k+8}=e<a_{k+s+1}$. Since $S$ is of negative excess weight, $b>e$, and we see that $T=U V(a, 1, r)\left(v_{e}\right)^{s}$ also has negative excess weight; i.e. $b(s+r)>w_{u}+a_{1}+\cdots+a_{r}+r$ $+s e$. Then $T^{*}=U u_{k} V(a, 1, k+s)$ has excess weight $w_{u}+k+a_{1}+\cdots$ $+a_{r}+e(s+k-r)+k+s-(b+1)(k+s)<(k-r)(e+1-b) \leqq 0$. But this is a contradiction since $T^{*}$, as a factor of $A$, cannot have negative excess weight.

Thus $S$ must involve a $v_{j}$ with $j \geqq a_{k}+1$ and we may assume that $S$, of signature $(m, n)$ and weight $w$, is equal to $V(a, 1, k) T(u, v)$. Now 
$S^{*}=V(a, 1, k) T\left(u, v_{1}\right) u_{k}$ is of signature $(m+1, n)$ and weight $w+n$ $-k+k=w+n$, and since $S^{*}$ is a factor of $A$, the weight of $S^{*}$ $\geqq(m+1) n$. That is, $w+n \geqq(m+1) n$ or $w \geqq m n$. This contradicts our assumption that $S$ was of negative excess weight and consequently there is no such factor of $Q$. Using the symmetry of [uv], this completes the proof of the theorem of this paper.

To obtain the stronger result of Lemma II, if $A$ involves $v_{0}$, we interchange the roles of $u$ and $v$ and find $A \equiv(-1)^{r} c v^{d_{2}} u^{d_{1}}$ where $c>0$ and $r$ is the $v$-weight of $A$. By the theorem of [3],

$$
A \equiv(-1)^{r+d_{1} d_{2}} c u^{d_{1} d_{d_{1}}},
$$

and since $A$ is of excess weight zero, (u-weight of $A)+(v$-weight of $A$ ) $=t+r=d_{1} d_{2}$. Thus $(-1)^{r+d_{1} d_{2}}=(-1)^{t}$.

\section{BIBLIOGRAPHY}

1. H. Levi, On the structure of differential polynomials and on their theory of ideals, Trans. Amer. Math. Soc. 51 (1942), 532-568.

2. D. G. Mead, Differential ideals, Proc. Amer. Math. Soc. 6 (1955), 420-432.

3. - A note on the ideal [uv], Proc. Amer. Math. Soc. 14 (1963), 607-608.

4. Kathleen B. O'Keefe, On a problem of J. F. Ritt, unpublished paper.

5. J. F. Ritt, Differential algebra, Amer. Math. Soc. Colloq. Publ. Vol. 33, Amer. Math. Soc., Providence, R. I., 1950.

University of Santa Clara 